\title{
Topological Phase Transitions in the Golden String-Net Model
}

\author{
Marc Daniel Schulz, ${ }^{1,2}$ Sébastien Dusuel, ${ }^{3}$ Kai Phillip Schmidt, ${ }^{1}$ and Julien Vidal ${ }^{2}$ \\ ${ }^{1}$ Lehrstuhl für Theoretische Physik I, Technische Universität Dortmund, \\ Otto-Hahn-Straße 4, 44221 Dortmund, Germany \\ ${ }^{2}$ Laboratoire de Physique Théorique de la Matière Condensée, CNRS UMR 7600, \\ Université Pierre et Marie Curie, 4 Place Jussieu, 75252 Paris Cedex 05, France \\ ${ }^{3}$ Lycée Saint-Louis, 44 Boulevard Saint-Michel, 75006 Paris, France
}

\begin{abstract}
We examine the zero-temperature phase diagram of the two-dimensional Levin-Wen string-net model with Fibonacci anyons in the presence of competing interactions. Combining high-order series expansions around three exactly solvable points and exact diagonalizations, we find that the non-Abelian doubled Fibonacci topological phase is separated from two nontopological phases by different second-order quantum critical points, the positions of which are computed accurately. These trivial phases are separated by a first-order transition occurring at a fourth exactly solvable point where the ground-state manifold is infinitely many degenerate. The evaluation of critical exponents suggests unusual universality classes.

PACS numbers: 71.10.Pm, 75.10.Jm, 03.65.Vf, 05.30.Pr
\end{abstract}

Quantum phases of matter are often well described by local order parameters and Landau-Ginzburg symmetrybreaking theory is an efficient tool to analyze transitions between these phases. However, in the late 1980s, a new class of phases that cannot be understood in terms of local symmetries has emerged in the context of high-temperature superconductivity [1-3]. These phases, dubbed topological because of their sensitivity to the system topology, have stimulated many studies in different domains (see Ref. 4 for a recent review). One of the most intriguing properties of topologically ordered phases is that they are robust against local (not too strong) perturbations $[5,6]$. This stability makes them especially appealing for quantum computation [7] as well as good candidates for quantum memories [8]. Several experiments have been proposed to realize the so-called topologically protected qubits [9]. In this perspective, a theoretical characterization of the robustness of topological phases under strong perturbations as well as the nature of the phase transitions signaling their breakdown is undoubtedly an important issue. Thanks to recently proposed exactly solvable lattice models realizing various topological phases of matter $[5,10,11]$, this program has been undertaken for several models [12-25].

The main purpose of this Letter is to go one step beyond, by studying the phase diagram of a paradigmatic 2D non-Abelian model. We consider the LevinWen model [11] on the honeycomb lattice with Fibonacci anyons (the golden string-net model) in the presence of the same perturbation as the one introduced in Ref. 20 . We determine the extension of the doubled Fibonacci (DFib) topological phase and show that it is separated from two other nontopological phases via second-order transitions that are analyzed in detail. Hilbert space - Following the Levin-Wen construction, we consider a honeycomb lattice with anyonic degrees of freedom living on its edges. In the Fibonacci string-net model, these local (microscopic) degrees of freedom can be in two different states $|0\rangle$ or $|1\rangle$. The Hilbert space $\mathcal{H}$ is restricted to states that satisfy the so-called branching rules stemming from the non-Abelian fusion rules

$$
\begin{aligned}
0 \times a=a \times 0 & =a \text { for } a \in\{0,1\}, \\
1 \times 1 & =0+1 .
\end{aligned}
$$

At each vertex of the honeycomb lattice, the fusion rules must not be violated; i.e., if one edge is in state $|1\rangle$, then at least one of the two other edges must be in the same state. For an arbitrary trivalent graph with $N_{\mathrm{v}}$ vertices, the dimension of the Hilbert space is then given by

$$
\operatorname{dim} \mathcal{H}=\left(1+\varphi^{2}\right)^{\frac{N_{\mathrm{v}}}{2}}+\left(1+\varphi^{-2}\right)^{\frac{N_{\mathrm{v}}}{2}},
$$

where $\varphi=\frac{1+\sqrt{5}}{2}$ is the golden ratio (see, e.g., Ref. 26).

Model. - We study the following Hamiltonian [20],

$$
H=-J_{\mathrm{p}} \sum_{p} \delta_{\Phi(p), 0}-J_{\mathrm{e}} \sum_{e} \delta_{l(e), 0} .
$$

The first term is the string-net Hamiltonian introduced by Levin and Wen [11]. It involves the projector $\delta_{\Phi(p), 0}$ onto states with no flux $\Phi(p)$ through plaquette $p[11,20]$. The second term is diagonal in the basis introduced above since $\delta_{l(e), 0}$ is the projector onto state $|0\rangle$ on edge $e$.

To help the reader to grasp the physical content of this Hamiltonian, let us mention what happens if one replaces Eq. (2) by the simpler Abelian $\mathbb{Z}_{2}$ fusion rule $1 \times 1=0$. The model then becomes Kitaev's toric code [5] on the honeycomb lattice, restricted to the charge-free sector (because of the branching rules), in the presence of a magnetic field $J_{\mathrm{e}}$ in the $x$ direction. Indeed, one can write the Hamiltonian in terms of Pauli matrices, with $\delta_{\Phi(p), 0}=\left(\mathbb{1}+\prod_{e \in p} \sigma_{e}^{z}\right) / 2$ and $\delta_{l(e), 0}=\left(\mathbb{1}+\sigma_{e}^{x}\right) / 2$. Identifying plaquette fluxes with spin $1 / 2$ variables as done in Refs. 12 and 13 for the square lattice, the Hamiltonian can further be mapped onto the transverse-field 
Ising model on the triangular lattice (of plaquettes), with coupling $J_{\mathrm{e}}$ and transverse-field $J_{\mathrm{p}}$.

Limiting cases. - For convenience, let us set $J_{\mathrm{p}}=\cos \theta$ and $J_{\mathrm{e}}=\sin \theta$. To our knowledge, contrary to the ladder geometry studied in Ref. 20, the Hamiltonian $H$ is exactly solvable only at the four points for which $\theta$ is a multiple of $\pi / 2$. In the following, we discuss the lowenergy spectrum of $H$ as well as the corresponding phases around these special points and give some arguments in favor of transitions between them.

(a) $\theta=0$ : For $J_{\mathrm{p}}>0$ and $J_{\mathrm{e}}=0$, the model reduces to the golden string-net model. Ground states $|\mathrm{g}\rangle$ are fluxfree states satisfying $\delta_{\Phi(p), 0}|\mathrm{~g}\rangle=|\mathrm{g}\rangle$ for all $p$ and thus have an energy per plaquette $e_{0}=E_{0} / N_{\mathrm{p}}=-1\left(N_{\mathrm{p}}\right.$ being the number of plaquettes). Their degeneracy depends on the system topology which is the most salient property of a topological phase. For the Fibonacci string-net model on any trivalent graph, the ground state is unique on a sphere [27] whereas it is fourfold degenerate on a torus. Interestingly, one can also compute the degeneracy of the $k$ th excited states (with energy $E_{k}=E_{0}+k$ )

$$
\mathcal{D}_{k}=\left(\begin{array}{c}
N_{\mathrm{p}} \\
k
\end{array}\right)\left(p F_{k-1}^{2}+q F_{k}^{2}+r F_{k} F_{k-1}\right),
$$

where we introduced the famous Fibonacci sequence defined for any integer $n$ by $F_{n+1}=F_{n}+F_{n-1}$, with $F_{-1}=1$ and $F_{0}=0$. The integers $(p, q, r)$ depend on the surface considered. For instance, one has $(p, q, r)=(1,0,0)$ on a sphere whereas $(p, q, r)=(4,1,4)$ on a torus. Equation (5) shows that for the Fibonacci theory, an odd number of excitations can exist on a compact surface contrary to the charge-free toric code discussed above where fluxes are always created and annihilated by pairs. Note that the binomial coefficient simply arises from the different ways to choose $k$ plaquettes carrying the flux excitations among $N_{\mathrm{p}}$.

Products of $t w o F_{k}$ 's stem from the fact that the "emergent" flux excitations are not the microscopic Fibonacci anyons but are achiral combinations of two Fibonacci anyons (details will be given in Ref. 28). The non-Abelian topological phase in the vicinity of $\theta=0$ is described by a DFib theory [11, 29-33]. Excitations have a trivial topological spin [11] and can fuse to the vacuum (also called trivial particle) [34]. As such, they can also be considered as bosons [35] and hence condense.

(b) $\theta=\pi$ : For $J_{\mathrm{e}}=0$ and $J_{\mathrm{p}}<0$, the low-energy spectrum is very different. Indeed, in this case, the groundstate manifold is $\mathcal{D}_{N_{\mathrm{p}}}$-fold degenerate and spanned by all states $|g\rangle$ satisfying $\delta_{\Phi(p), 0}|\mathrm{~g}\rangle=0$ for all $p$. As discussed above, this degeneracy depends on the topology through its indices $(p, q, r)$ so that one might be tempted to consider the system as topologically ordered. However, the local operator $\sum_{e} \delta_{l(e), 0}$ couples the ground states and splits the degeneracy for any $J_{\mathrm{e}} \neq 0$. As a consequence, the system cannot be considered as topologically ordered [36].

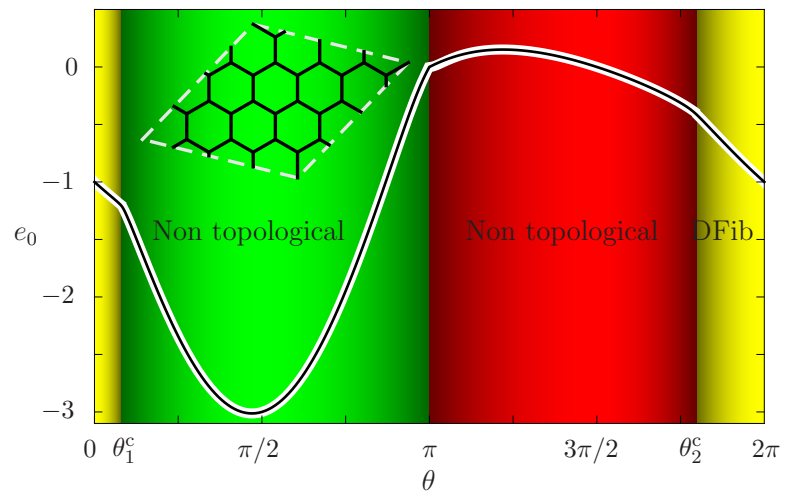

FIG. 1. (color online). Ground-state energy per plaquette $e_{0}=E_{0} / N_{\mathrm{p}}$ as a function of $\theta$. ED results (black line) for $N_{\mathrm{p}}=\sqrt{13} \times \sqrt{13}$ plaquettes (see inset) are in excellent agreement with typical Padé approximants (white lines) computed from high-order series expansions (see Supplemental Material).

Owing to this huge degeneracy, we have not been able to analyze the vicinity of this point. However, numerical results obtained by exact diagonalizations clearly show that $(i)$ the degeneracy is lifted as soon as the coupling $J_{\mathrm{e}} \neq 0$ and $(i i)$ the ground state for $\theta=\pi^{ \pm}$is unique and adiabatically connected to the polarized ground states found at $\theta=\pi / 2$ and $3 \pi / 2$, respectively, (see discussion below). This result is in stark contrast with the scenario described in Ref. 20 on the ladder where a gapless phase is observed for $\theta \in[\pi, 3 \pi / 2]$. In addition, as can be seen in Fig. 3 (central panel), we found a jump in $\partial_{\theta} e_{0}$ at $\theta=\pi$ (for all system sizes) indicating that the two gapped phases $\left(\theta=\pi^{+}\right.$and $\left.\theta=\pi^{-}\right)$are separated by a first-order phase transition.

(c) $\theta=\pi / 2:$ For $J_{\mathrm{p}}=0$, the Hamiltonian $H$ is diagonal in the canonical basis of states satisfying the branching rules. For $J_{\mathrm{e}}>0$, the ground state is unique whatever the topology and corresponds to the fully polarized state where all edges are in the state $|0\rangle$ (with eigenenergy $E_{0}=-N_{\mathrm{e}}$, where $N_{\mathrm{e}}$ is the total number of edges). First excited states are obtained by flipping six links around one hexagon. They behave as trivial hard-core bosons that become dynamical when the coupling $J_{\mathrm{p}}$ is switched on. Thus, near $\theta=\pi / 2$, the system is gapped but not topologically ordered, making the occurrence of a phase transition in the interval $[0, \pi / 2]$ compulsory.

(d) $\theta=3 \pi / 2$ : For $J_{\mathrm{e}}<0$ and $J_{\mathrm{p}}=0$, the Hamiltonian is also diagonal and the unique ground state is the fully polarized state where all edges are in the states $|1\rangle\left(e_{0}=0\right)$. Note that such a state would be forbidden by the Abelian $\mathbb{Z}_{2}$ fusion rules. First excited states are obtained from the ground state by flipping a single link. As previously, these localized excitations are trivial hardcore bosons that become mobile when $J_{\mathrm{p}} \neq 0$ so that one expects a phase transition in the interval $[3 \pi / 2,2 \pi]$.

Phase diagram. - To determine the zero-temperature 


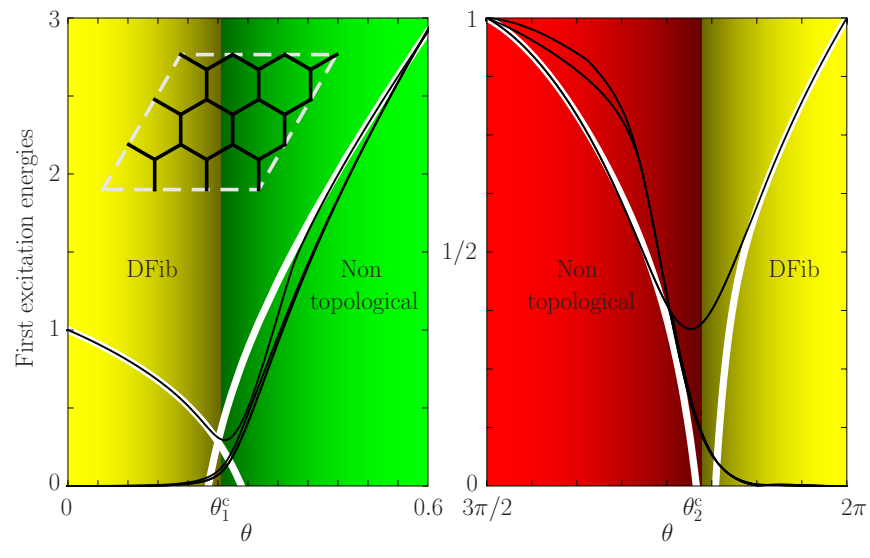

FIG. 2. (color online). First four excitation energies obtained from the ED results (black lines) for $N_{\mathrm{p}}=3 \times 3$ plaquettes compared with the low-energy gap computed from high-order bare series expansions (white lines) given in the Supplemental Material. The topological degeneracy splitting is clearly observed in the vicinity of the critical points. For symmetry reasons, this splitting is only partial for the system considered here (see inset).

phase diagram, we combined two different approaches. First, we performed high-order series expansions in the thermodynamical limit by means of several methods [37$39]$, around the exactly solvable points $\theta=0, \pi / 2$, and $3 \pi / 2$ described above. This yields the ground-state energy per plaquette $e_{0}$ as well as the quasiparticle dispersion from which the low-energy gap $\Delta$ can be extracted. Lengthy expressions of these series expansions can be found in the Supplemental Material. This method allows one to accurately compute the critical couplings for which the gap vanishes. These points are associated to second-order transitions but might not be relevant if first-order transitions are present (see Refs. 17 and 19 for details about this issue in a similar context). Second, we perform exact diagonalizations (ED) for lattices with periodic boundary conditions. As can be seen in Figs. 1 and 2, series expansions and ED data are in very good agreement except in the vicinity of the transition points where finite-order and finite-size effects are important.

Combining these two methods (ED and series expansions) we found that the DFib topological phase near $\theta=0$ ranges from $\theta_{2}^{\mathrm{c}} \simeq-0.63(=5.65)$ to $\theta_{1}^{\mathrm{c}} \simeq 0.255$. As we shall now argue, we associate these two points to second-order transitions. The first piece of evidence pleading in favor of such a scenario follows from ED and is the behavior of $\partial_{\theta}^{2} e_{0}$ that clearly decreases with the system size near these points (see left and right panels in Fig. 3). In addition, the position of the low-energy gap minimum as well as the topological degeneracy splitting shown in Fig. 2 lie in the same region as the position of the minimum of $\partial_{\theta}^{2} e_{0}$. Let us also note that we did not find any relevant level crossing in the excitation spectrum that could lead to a first-order transition. The sec- ond argument comes from the high-order perturbation theory. As can be seen in the Supplemental Material, the series behave very differently for positive and negative $J_{\mathrm{e}}$. So, let us first discuss the most favorable case $J_{\mathrm{e}}>0\left(J_{\mathrm{p}}>0\right)$. A close inspection of the series expansion near $\theta=0$ and $\theta=\pi / 2$ reveals three important features: (i) the sign of each coefficient is the same in all series; (ii) series of the ground-state energy intersect in two order-dependent points - the possible merging of these points, in the infinite-order limit, being a signal of a second-order transition since series then have to be tangential at the critical point; (iii) series of the gap intersect in a unique (still order-dependent) point, see, e.g., the left part of Fig. 2. We emphasize that the value of the gap at this crossing point decreases when the order increases and eventually vanishes in the infinite-order limit. In Fig. 4, we plotted the position of these crossing points as a function of the (inverse) order as well as the position of the minimum of the low-energy gap and of $\partial_{\theta}^{2} e_{0}$ as a function of $N_{\mathrm{p}}^{-1}$ computed from ED results. As can be seen, all data seem to converge to the same point $\theta_{1}^{\mathrm{c}} \in[0.255,0.256]$ in the infinite-order (size) limit, providing a smoking-gun evidence of a second-order transition.

Unfortunately, the case $J_{\mathrm{e}}<0\left(J_{\mathrm{p}}>0\right)$ is more involved for three reasons. First, series expansions of $e_{0}$ and $\Delta$ in this region have alternate signs so that the previous criteria based on crossing points cannot be used. Second, contrary to the case $J_{\mathrm{e}}>0$, the momentum minimizing the dispersion of the low-energy quasiparticles is not at the $\Gamma$ point and only belongs to the reciprocal lattice of $3 p \times 3 q$ systems, $(p, q) \in \mathbb{N}^{2}$. The only system with such characteristics considered in this study is the $3 \times 3$ lattice so that one cannot perform any reliable
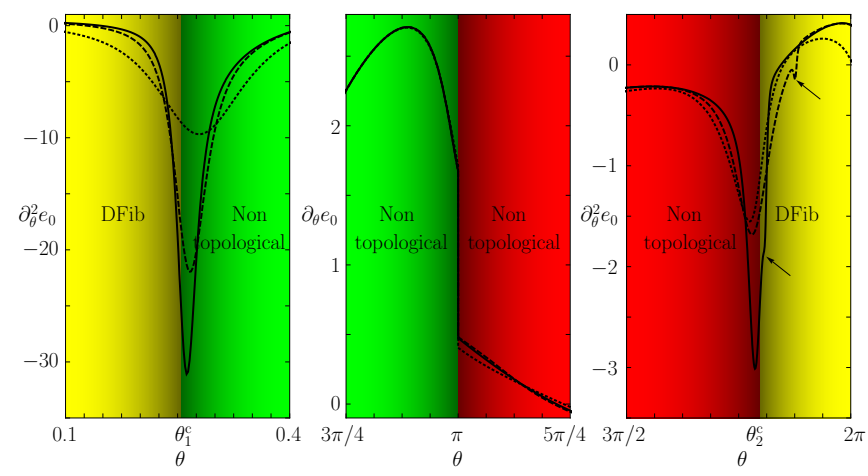

FIG. 3. (color online). ED results for $N_{\mathrm{p}}=2 \times 2$ (dotted line), $3 \times 3$ (dashed line), and $\sqrt{13} \times \sqrt{13}$ (solid line) plaquettes. Left and right panels : $\partial_{\theta}^{2} e_{0}$ decreases with the system size indicating second-order transitions at $\theta_{1}^{\mathrm{c}}$ and $\theta_{2}^{\mathrm{c}}$. Central panel : $\partial_{\theta} e_{0}$ displays a clear jump at $\theta=\pi$ indicating a first-order transition. Dips indicated by arrows in the DFib topological phase are due to (irrelevant and avoided) level crossings between the four lowest-energy levels that become degenerate in the thermodynamical limit on a torus. 


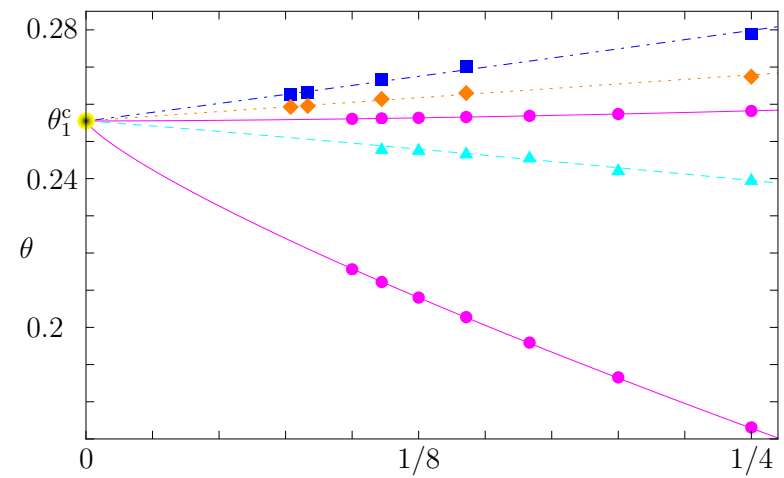

FIG. 4. (color online). Position of different quantities as a function of either the inverse order $n^{-1}$ of the corresponding series expansion [crossing points of $e_{0}$ (dots), crossing points of the gap (triangles)] or $N_{\mathrm{p}}^{-1}$ [minimum of the gap (squares), minimum of $\partial_{\theta}^{2} e_{0}$ (diamonds)]. Lines are power-law fits obtained by choosing $\theta_{1}^{\mathrm{c}}$ such that it maximizes the correlations for the crossing points of $e_{0}$ (solid lines) that are the most accurate results in this work. These crossing points have been obtained from order $n$ series around $\theta=0$ and order $2 n-1$ series around $\theta=\pi / 2$. For the crossing points of the gap, we used order $n$ series around both limits.

analysis from ED data near $\theta_{2}^{\mathrm{c}}$. Third, because of the nature of the low-energy states, maximum orders reachable around $\theta=3 \pi / 2$ are smaller than around $\theta=\pi / 2$ (see Supplemental Material). Nevertheless, using the gap series expansion around $\theta=0$, it is possible to perform (dlogPadé) resummations that lead to a position of the critical point $\theta_{2}^{c} \in[5.61,5.62]$. Note that the same methods for $J_{\mathrm{e}}>0$ would lead to $\theta_{1}^{\mathrm{c}} \in[0.259,0.261]$. This value differs from the one proposed above only by a few percent and it overestimates the extension of the topological DFib phase. Consequently, as we have no alternative approach, we roughly estimate that $\theta_{2}^{\mathrm{c}}$ lies in the range $[5.6,5.7]$.

Critical exponents. - The obvious question that arises next concerns universality classes associated to the transition points. In the absence of a local order parameter, the only meaningful critical exponents for topological phase transitions are those associated to the spectrum. Let us remind that for a second-order transition, the gap vanishes, at large linear system size $L$ and at the critical point, as $\Delta \sim L^{-z}$ where $z$ is the dynamical exponent. In the thermodynamical limit, one further has $\Delta \sim\left|\theta-\theta^{\mathrm{c}}\right|^{z \nu}$ and $\partial_{\theta}^{2} e_{0} \sim\left|\theta-\theta^{\mathrm{c}}\right|^{-\alpha}$.

As already explained, ED are only useful quantitatively around $\theta_{1}^{c}$ although restricted to systems of small sizes. Using the gap data, we find $z \simeq 1.2$. A finite-size analysis of $e_{0}$ yields a surprisingly good data collapse for the $3 \times 3$ and $\sqrt{13} \times \sqrt{13}$ systems, with $\theta_{1}^{\text {c }} \simeq 0.255, z \simeq 1, \nu \simeq 0.4$ and $\alpha \simeq 0.8$ [28]. The rather large value of $\alpha$ might be responsible for the quality of the data collapse. We emphasize that these values are compatible with the previous estimate of $\theta_{1}^{\mathrm{c}}$ as well as with the hyperscaling relation $2-\alpha=\nu(2+z)$. The above exponents are furthermore in agreement with dlogPadé resummations of the series expansion around $\theta=0$ which yield $z \nu \in[0.4,0.44]$. We discard results from resummations around $\theta=\pi / 2$ because these results are not as well converged, since for a given order, clusters needed to compute series around this point are twice as small as those needed around $\theta=0$. Note that as usual, extracting $\alpha$ from series of $e_{0}$ does not give any conclusive result. Concerning the critical behavior at $\theta_{2}^{\mathrm{c}}$, we only use dlogPadé resummation around $\theta=0$ and we obtain a gap exponent $z \nu \in[0.56,0.58]$.

Outlook. - It is difficult to provide some error bars concerning these values. To estimate these errors, we performed similar series expansion analysis for the Fibonacci ladder (for which exponents are known exactly [20]) and for the $2 \mathrm{D} \mathbb{Z}_{2}$ string-net model (having either Ising or $X Y$ transitions depending on the sign of $J_{\mathrm{e}}$ ). The results we obtained [28] lead us to conclude that critical exponents are to be considered with a precision of about $10 \%$. As a conclusion, we found two different second-order transitions with universality classes that, to the best of our knowledge, are as yet unknown in the context of topological phase transitions. Let us mention that a critical DFib wave function has been proposed [40] but its relevance for the present problem requires further studies [28].

To gain more understanding about these transitions, different approaches could be used, e.g., variational methods or Monte Carlo simulations, although a naive implementation of the latter should suffer from the sign problem. It would also be worth studying similar models with a DFib phase $[40,41]$ as well as different topological phases. Finally, another important issue concerns transitions between two distinct topological phases [35]. Given the ubiquity of Fibonacci anyons in many different physics domain such as topological quantum computation, condensed matter, or atomic physics [42], we hope that the present work will stimulate such investigations.

We thank K. Coester, B. Douçot, M. Kamfor, and J.-B. Zuber for fruitful discussions. K. P. Schmidt acknowledges ESF and EuroHorcs for funding through his EURYI.

[1] X.-G. Wen, F. Wilczek, and A. Zee, Phys. Rev. B 39, 11413 (1989).

[2] X.-G. Wen, Phys. Rev. B 40, 7387 (1989).

[3] X.-G. Wen, Int. J. Mod. Phys. B 4, 239 (1990).

[4] X.-G. Wen, arXiv:1210.1281.

[5] A. Y. Kitaev, Ann. Phys. 303, 2 (2003).

[6] S. Bravyi, M. B. Hastings, and S. Michalakis, J. Math. Phys. 51, 093512 (2010).

[7] See http://www.theory.caltech.edu/people/preskill/ph219/ for a pedagogical introduction.

[8] E. Dennis, A. Kitaev, A. Landahl, and J. Preskill, J. 
Math. Phys. 43, 4452 (2002).

[9] B. Douçot and L. B. Ioffe, Rep. Prog. Phys. 75, 072001 (2012).

[10] X.-G. Wen, Phys. Rev. Lett. 90, 016803 (2003).

[11] M. A. Levin and X.-G. Wen, Phys. Rev. B 71, 045110 (2005).

[12] S. Trebst, P. Werner, M. Troyer, K. Shtengel, and C. Nayak, Phys. Rev. Lett. 98, 070602 (2007).

[13] A. Hamma and D. A. Lidar, Phys. Rev. Lett. 100, 030502 (2008).

[14] J. Yu, S.-P. Kou, and X.-G. Wen, Europhys. Lett. 84, 17004 (2008).

[15] J. Vidal, S. Dusuel, and K. P. Schmidt, Phys. Rev. B 79, 033109 (2009).

[16] J. Vidal, R. Thomale, K. P. Schmidt, and S. Dusuel, Phys. Rev. B 80, 081104 (2009).

[17] S. Dusuel, M. Kamfor, R. Orús, K. P. Schmidt, and J. Vidal, Phys. Rev. Lett. 106, 107203 (2011).

[18] F. Wu, Y. Deng, and N. Prokof'ev, Phys. Rev. B 85, 195104 (2012).

[19] M. D. Schulz, S. Dusuel, R. Orús, J. Vidal, and K. P. Schmidt, New J. Phys. 14, 025005 (2012).

[20] C. Gils, S. Trebst, A. Kitaev, A. W. W. Ludwig, M. Troyer, and Z. Wang, Nat. Phys. 5, 834 (2009).

[21] C. Gils, J. Stat. Mech. P07019 (2009).

[22] A. W. W. Ludwig, D. Poilblanc, S. Trebst, and M. Troyer, New J. Phys. 13, 045014 (2011).

[23] D. Poilblanc, A. W. W. Ludwig, S. Trebst, and M. Troyer, Phys. Rev. B 83, 134439 (2011).

[24] M. H. Freedman, J. Gukelberger, M. B. Hastings, S. Trebst, M. Troyer, and Z. Wang, Phys. Rev. B 85, 045414 (2012).
[25] F. J. Burnell, S. H. Simon, and J. K. Slingerland, New J. Phys. 14, 015004 (2012).

[26] S. H. Simon and P. Fendley, J. Phys. A 46, 105002 (2013).

[27] Y. Hu, S. D. Stirling, and Y.-S. Wu, Phys. Rev. B 85, 075107 (2012).

[28] M. D. Schulz et al., (to be published).

[29] L. Fidkowski, M. Freedman, C. Nayak, K. Walker, and Z. Wang, Commun. Math. Phys. 287, 805 (2009).

[30] Z. Wang, Topological Quantum Computation, CBMS Regional Conference Series in Mathematics, Number 112 (2010).

[31] F. J. Burnell and S. H. Simon, Ann. Phys. 325, 2550 (2010).

[32] F. J. Burnell and S. H. Simon, New J. Phys. 13, 065001 (2011).

[33] F. J. Burnell, S. H. Simon, and J. K. Slingerland, Phys. Rev. B 84, 125434 (2011).

[34] R. Koenig, G. Kuperberg, and B. W. Reichardt, Ann. Phys. 325, 2707 (2010)

[35] F. A. Bais and J. K. Slingerland, Phys. Rev. B 79, 045316 (2009).

[36] Z. Nussinov and G. Ortiz, Ann. Phys. 324, 977 (2009).

[37] P.-O. Löwdin, J. Math. Phys. 3, 969 (1962).

[38] M. Takahashi, J. Phys. C 10, 1289 (1977).

[39] C. Knetter and G. S. Uhrig, Eur. Phys. J. B 13, 209 (2000).

[40] P. Fendley, Ann. Phys. 323, 3113 (2008).

[41] P. Fendley, S. V. Isakov, and M. Troyer, arXiv:1210.5527.

[42] I. Lesanovsky and H. Katsura, Phys. Rev. A 86, 041601 (2012). 


\section{SUPPLEMENTAL MATERIAL}

In the following, we give the series expansions in the different phases for the ground-state energy per plaquette $e_{0}$ and the quasiparticle gaps $\Delta^{ \pm}$, for positive and negative signs of the dimensionless parameter $t=J_{\mathrm{e}} / J_{\mathrm{p}}=\tan \theta$ respectively. For the sake of clarity, we give below the numerical values of the coefficients with 16 digits.

\section{Expansions in the vicinity $\theta=0$}

The ground-state energy per plaquette $e_{0}$ near $\theta=0\left(J_{\mathrm{p}}=1, J_{\mathrm{e}}=0\right)$ has been obtained up to order 11 using operator perturbation theory [38], whereas quasiparticle gaps $\Delta^{ \pm}$were obtained up to order 9 using perturbative continuous unitary transformations. [39]

$$
\begin{aligned}
e_{0} / J_{\mathrm{p}}= & -1-0.8291796067500631 t-0.3 t^{2}-0.2329179606750063 t^{3}-0.3758359213500126 t^{4} \\
& -0.6934622369921362 t^{5}-1.517757831138397 t^{6}-3.615896887905089 t^{7}-9.257482947753094 t^{8} \\
& -24.89646210135949 t^{9}-69.63655938933877 t^{10}-200.8253697230269 t^{11} \\
\Delta^{+} / J_{\mathrm{p}}=1- & 1.658359213500126 t-2.029179606750063 t^{2}-3.107113095525145 t^{3}-8.042597266963313 t^{4} \\
& -19.16249885423558 t^{5}-58.31720409052607 t^{6}-164.4421257647495 t^{7}-528.5318111014412 t^{8} \\
& -1615.453328025113 t^{9} \\
\Delta^{-} / J_{\mathrm{p}}= & +0.8291796067500631 t+0.1145898033750315 t^{2}+0.5110332556124590 t^{3}+0.4044760408194119 t^{4} \\
& +0.9554382719956335 t^{5}+1.784752477740017 t^{6}+4.523961920423115 t^{7}+11.17294663306187 t^{8} \\
& +31.20020681009114 t^{9} .
\end{aligned}
$$

\section{Expansions in the vicinity $\theta=\pi / 2$}

The ground-state energy per plaquette $e_{0}$ near $\theta=\pi / 2\left(J_{\mathrm{e}}=1, J_{\mathrm{p}}=0\right)$ has been obtained up to order 19 using a partitioning technique provided by Löwdin [37], whereas quasiparticle gaps $\Delta^{ \pm}$were obtained up to order 11 using operator perturbation theory [38] on appropriate periodic clusters.

$$
\begin{aligned}
e_{0} / J_{\mathrm{e}}= & -3-2.763932022500210 \cdot 10^{-1} t^{-1}-3.333333333333333 \cdot 10^{-2} t^{-2}-2.484519974999766 \cdot 10^{-3} t^{-3} \\
& -1.473090114646592 \cdot 10^{-4} t^{-4}-1.762516450320833 \cdot 10^{-5} t^{-5}-3.114829150546602 \cdot 10^{-6} t^{-6} \\
& -4.974954832303385 \cdot 10^{-7} t^{-7}-8.712942025753695 \cdot 10^{-8} t^{-8}-1.680470831303724 \cdot 10^{-8} t^{-9} \\
& -3.252798060742993 \cdot 10^{-9} t^{-10}-6.452247707667803 \cdot 10^{-10} t^{-11}-1.328942917494399 \cdot 10^{-10} t^{-12} \\
& -2.786531244027440 \cdot 10^{-11} t^{-13}-5.923931315552463 \cdot 10^{-12} t^{-14}-1.280290681522505 \cdot 10^{-12} t^{-15} \\
& -2.803432623420322 \cdot 10^{-13} t^{-16}-6.202995686227467 \cdot 10^{-14} t^{-17}-1.386248233596245 \cdot 10^{-14} t^{-18} \\
& -3.125540962122164 \cdot 10^{-15} t^{-19} \\
& -1.826128841179490 \cdot 10^{-3} t^{-4}-2.141218566177655 \cdot 10^{-4} t^{-5}-7.057273308076495 \cdot 10^{-5} t^{-6} \\
& -7.713120260517609 \cdot 10^{-6} t^{-7}-1.822619200222926 \cdot 10^{-6} t^{-8}-1.739394034185955 \cdot 10^{-7} t^{-9} \\
& -1.068512192171555 \cdot 10^{-7} t^{-10}-1.583732801424164 \cdot 10^{-8} t^{-11}
\end{aligned}
$$


Expansions in the vicinity $\theta=3 \pi / 2$

The ground-state energy per plaquette $e_{0}$ near $\theta=3 \pi / 2\left(J_{\mathrm{e}}=-1, J_{\mathrm{p}}=0\right)$ has been obtained up to order 9 using partitioning techniques [37], whereas quasiparticle gaps $\Delta^{ \pm}$were obtained up to order 6 using perturbative continuous unitary transformations. [39]

$$
\begin{aligned}
e_{0} /\left(-J_{\mathrm{e}}\right)= & 3.013155617496425 \cdot 10^{-1} t^{-1}-1.132044933254820 \cdot 10^{-1} t^{-2}+2.807797460719963 \cdot 10^{-2} t^{-3} \\
& -4.507809490972001 \cdot 10^{-3} t^{-4}-3.037988446794379 \cdot 10^{-3} t^{-5}+4.596752532754356 \cdot 10^{-3} t^{-6} \\
& -1.633669374413878 \cdot 10^{-3} t^{-7}-2.288717115668441 \cdot 10^{-3} t^{-8}+3.812642130493073 \cdot 10^{-3} t^{-9}, \\
\Delta^{+} /\left(-J_{\mathrm{e}}\right)=1 & -7.331262919989905 \cdot 10^{-1} t^{-1}+2.627515502196252 \cdot 10^{-1} t^{-2}-1.016556730679853 \cdot 10^{-1} t^{-3} \\
& +1.966652521926989 \cdot 10^{-2} t^{-4}-7.070423645089451 \cdot 10^{-2} t^{-5}+1.098516898950685 \cdot 10^{-1} t^{-6}, \\
\Delta^{-} /\left(-J_{\mathrm{e}}\right)=1 & +2.668737080010095 \cdot 10^{-1} t^{-1}-2.048165955279984 \cdot 10^{-1} t^{-2}-7.571315893653220 \cdot 10^{-2} t^{-3} \\
& +3.964262957878962 \cdot 10^{-2} t^{-4}+7.257956045336907 \cdot 10^{-2} t^{-5}-6.691149658050659 \cdot 10^{-2} t^{-6} .
\end{aligned}
$$

